\title{
Top Quark Physics
}

\author{
M. Jeżabek a b*

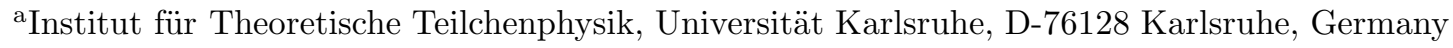 \\ ${ }^{\mathrm{b}}$ Institute of Nuclear Physics, ul.Kawiory 26a, PL-30055 Cracow, Poland
}

Top quark studies at future $e^{+} e^{-}$colliders are considered. Two issues are discussed: a - Some results are presented on the decays of top quarks. Energy distributions of charged leptons and neutrinos in $t \rightarrow b W \rightarrow b e^{+} \nu$ and jets in $t \rightarrow b W \rightarrow b \bar{d} u$ decays are sensitive to the structure of $t b W$ vertex. Distributions of charged leptons from top decays are particularly useful in polarization studies whereas neutrinos are sensitive to deviations from the Standard Model. $\mathrm{b}$ - Recent calculations are reviewed on the top quark pair production in $e^{+} e^{-}$annihilation. The differential cross sections in the threshold region can lead to an accurate determination of the top quark mass and the interquark potential. The effects of the top-Higgs Yukawa coupling and some higher order QCD corrections are also under control.

\section{TOP QUARK AT $\mathrm{e}^{+} \mathrm{e}^{-}$COLLIDERS}

Top quark is the heaviest fermion of the Standard Model. The existing direct lower mass limit|1] $m_{t} \geq 131 \mathrm{GeV}$ as well as the value $m_{t}=174_{-12-19}^{+11+17} \mathrm{GeV}$ derived from the recent fit to LEP+SLC data [2] indicate that its production is out of reach of LEP II. (Two weeks after this talk had been delivered the top quark saga culminated at the press conference at FNAL when evidence for $t \bar{t}$ production was announced by the CDF Collaboration [3]. The reported value of the top quark mass $m_{t}=174 \pm 10_{-19}^{+17} \mathrm{GeV}$ agrees very well with the indirect determination [2].)

It has been argued [- 1 that single top production may be observed for $m_{t}$ up to about 180 $\mathrm{GeV}$ if LEP II center-of-mass energy is pushed to $\sqrt{s}=210 \mathrm{GeV}$. However, a recent article [5] found a dramatic cancellation of contributions to the reaction $e^{+} e^{-} \rightarrow e^{-} \bar{\nu} t \bar{b}$. A similar destructive interference damps the single top production in $e \gamma$ collisions [5]. It is noteworthy, however, that this does not occur for $p \bar{p}$ and $p p$ collisions [6] where for large top masses the cross section of single top production becomes comparable to that of $t \bar{t}$ production. In Fig. 1 the cross sction of reaction $e^{+} e^{-} \rightarrow e^{-} \bar{\nu} t \bar{b}$ is shown for $\sqrt{s}=170$, 190 and $210 \mathrm{GeV}$ [5]. The solid lines correspond

\footnotetext{
${ }^{*}$ Work partly supported by KBN under contract 2P30225206 and by DFG under contract 436POL173193S.
}

to the complete set of Feynman diagrams, the dashed lines to the photon exchange subset and the dotted lines to the Weizsäcker-Williams approximation. It is evident that even for the integrated luminosity $\mathcal{L}=500 \mathrm{pb}^{-1}$ and $\sqrt{s}=210$ $\mathrm{GeV}$ one expects no events. Therefore, the top quark can be observed in $e^{+} e^{-}$collisions only if the Next Linear Collider is built. Comprehensive studies have been performed of the physics potential of a $500 \mathrm{GeV}$ center-of-mass energy collider. The results are summarized in [7 9]. Theoretical studies on the top quark are reviewed in [10 13$]$.

\section{TOP QUARK DECAY}

\subsection{Total decay rate}

Top quark is the first heavy quark whose mass can be measured to better than $1 \%$ precision at a future $e^{+} e^{-}$collider [14]. Therefore, measurements of its width can test not only the Standard Model at the Born level, but also the QCD radiative corrections which are about $-8.5 \%$. This is in contrast to $b$ and $c$ quarks, where uncertainties in the masses and non-perturbative effects preclude this possibility.

Being much heavier than the $W$ boson, the top quark decays dominantly into $b W$ final states. In the Standard Model with three quark-lepton families, which is assumed throughout, the modulus of the element $V_{t b}$ of 
Table 1

Top quark width as the function of $m_{t}$ including $\left(\Gamma_{t}\right)$ and without $\left(\Gamma_{n w}^{(0)}\right)$ the contributions of the QCD, non-zero $W$ boson width (WBW) and electroweak (EW) corrections.

\begin{tabular}{l|c|cccc}
\hline$m_{t}[\mathrm{GeV}]$ & $\Gamma_{t}[\mathrm{GeV}]$ & $\Gamma_{n w}^{(0)}[\mathrm{GeV}]$ & $\mathrm{QCD}[\%]$ & WBW[\%] & EW[\%] \\
\hline 150.0 & 0.809 & 0.885 & -8.47 & -1.69 & 1.57 \\
160.0 & 1.033 & 1.130 & -8.49 & -1.60 & 1.62 \\
170.0 & 1.287 & 1.405 & -8.49 & -1.52 & 1.67 \\
180.0 & 1.572 & 1.714 & -8.48 & -1.45 & 1.70 \\
190.0 & 1.890 & 2.059 & -8.47 & -1.39 & 1.73 \\
200.0 & 2.242 & 2.440 & -8.46 & -1.33 & 1.76 \\
\hline
\end{tabular}

$y=\left(m_{\mathrm{w}} / m_{t}\right)^{2}$

$\mathcal{F}_{0}(y)=2(1-y)^{2}(1+2 y)$

and 16

$$
\begin{array}{r}
\mathcal{F}_{1}(y)=\mathcal{F}_{0}(y)\left[\frac{2}{3} \pi^{2}+4 \mathrm{Li}_{2}(y)+2 \ln y \ln (1-y)\right] \\
-(1-y)\left(5+9 y-6 y^{2}\right)+4 y\left(1-y-2 y^{2}\right) \ln y \\
+2(1-y)^{2}(5+4 y) \ln (1-y)
\end{array}
$$

The one loop electroweak corrections to the total decay rate have been calculated in the narrow width approximation [17]. They have turned out to be positive and rather small (1-2\%). A reason for this is that the contribution from the Higgs-top quark Yukawa coupling remains very small for realistic top quark masses [18]. The effect of the non-zero $W$ width is comparable in size to the electroweak correction but of the opposite sign [19]. Formulae for the QCD corrected total decay rate including non-zero $b$ quark mass and $W$ boson width are given in 16,19 . In Table [1 we present the results [19] for the total decay rate $\Gamma_{t}$ and its narrow width Born approximation $\Gamma_{n w}^{(0)}$. We give also the Standard Model contributions to the width of the top quark from the first order QCD corrections, $W$ boson width (WBW) and the electroweak (EW) corrections. A number of intrinsic uncertainties remains. It should be noted that the size of the electroweak corrections is comparable to the uncertainties from the as yet uncalculated $\mathcal{O}\left(\alpha_{s}{ }^{2}\right)$ correction. The present uncertainty in $\alpha_{s}$ and the ignorance concerning the second order QCD correction limit the accuracy of the prediction to about 1-2\%. Experimental errors as well as theoretical uncertanties in the 
determination of the top mass (c.f. Sect.3) can also lead to effects of similar magnitude.

\subsection{Energy distributions}

Figure 2. Distribution of $W$ energy for $m_{t}=174$ $\mathrm{GeV}$ without (dashed line) and with (solid line) QCD corrections

\subsubsection{Energy of W}

The dominance of the two-body $t \rightarrow b W$ decay implies that the energy of $W$ is strongly peaked. In the top quark rest frame the two-body kinematics implies

$E_{\mathrm{w}}=\frac{m_{t}^{2}+m_{\mathrm{w}}^{2}-m_{b}^{2}}{2 m_{t}}$

The effects of non-zero $W$ width and QCD corrections have been studied in [20]. In Fig, 2 the energy distribution $\mathrm{d} \Gamma / \mathrm{d} E_{\mathrm{w}}$ is shown as the function of $E_{\mathrm{w}}$ for $m_{t}=174 \mathrm{GeV}$. The Dirac delta spike in $E_{\mathrm{w}}$ is smeared by the effects of non-zero $W$ width, see dashed line. QCD corrections result in further distortion of the spectrum (solid line) because hard gluon radiation leads to a long radiative tail and the virtual corrections significantly reduce the height of the peak.

\subsubsection{Energy distributions of leptons}

The energy spectra of the leptons from semileptonic decays of the top quark are sensitive to $\mathrm{V}-\mathrm{A}$ form of $t b W$ vertex. In first order QCD approximation neglecting the masses of $b$ quark and the leptons one derives the following normalized energy distributions of the charged leptons and the neutrinos:

$\mathrm{A}_{\mathrm{l}}\left(x_{\ell}\right)=\frac{\mathrm{d} N}{\mathrm{~d} x_{\ell}}=12 \frac{\mathrm{F}_{0}^{+}\left(x_{\ell}, y\right)-a_{s} \mathrm{~F}_{1}^{+}\left(x_{\ell}, y\right)}{\mathcal{F}_{0}(y)-a_{s} \mathcal{F}_{1}(y)}$
$\mathrm{A}_{\nu}\left(x_{\nu}\right)=\frac{\mathrm{d} N}{\mathrm{~d} x_{\nu}}=12 \frac{\mathrm{F}_{0}^{-}\left(x_{\nu}, y\right)-a_{s} \mathrm{~F}_{1}^{-}\left(x_{\nu}, y\right)}{\mathcal{F}_{0}(y)-a_{s} \mathcal{F}_{1}(y)}$

for $y \leq x_{\ell, \nu} \leq 1$, where $x_{\ell, \nu}=2 E_{\ell, \nu} / m_{t}$ denote the scaled energies in the $t$ rest frame, the functions $\mathcal{F}_{0}(y)$ and $\mathcal{F}_{1}(y)$ have been defined in eqs.(4) and (5),

$\mathrm{F}_{0}^{+}(x, y)=x(1-x)$
$\mathrm{F}_{0}^{-}(x, y)=(x-y)(1-x+y)$

and 21,22

$$
\begin{aligned}
& \mathrm{F}_{1}^{+}(x, y)=\mathrm{F}_{0}^{+}(x, y) \Phi_{0}+x \Phi_{1}-(3+2 x+y) \Phi_{2 \diamond 3} \\
& \quad+5(1-x) \Phi_{4}+\left(-2 x y+9 x-4 x^{2}-2 y-y^{2}\right) \Phi_{5} \\
& \quad+y(4-4 x-y+y / x) / 2 \\
& \mathrm{~F}_{1}^{-}(x, y)=\mathrm{F}_{0}^{-}(x, y) \Phi_{0}+\left(-2 x y+x+y+y^{2}\right) \Phi_{1} \\
& \quad+(-5+2 x-3 y) \Phi_{2 \diamond 3}+(5+4 x y-5 x+3 y- \\
& \left.\quad 5 y^{2}-2 y^{2} / x\right) \Phi_{4}+\left(6 x y+9 x-4 x^{2}-11 y-2 y^{2}\right. \\
& \left.\quad+2 y^{2} / x\right) \Phi_{5}+y(2+3 x-3 y-2 y / x) / 2
\end{aligned}
$$

where

$$
\begin{aligned}
& \Phi_{0}=\frac{\pi^{2}}{3}+2 \operatorname{Li}_{2}(x)+2 \operatorname{Li}_{2}(y / x)+\ln ^{2}\left(\frac{1-y / x}{1-x}\right) \\
& \Phi_{1}=\frac{\pi^{2}}{6}+\operatorname{Li}_{2}(y)-\operatorname{Li}_{2}(x)-\operatorname{Li}_{2}(y / x) \\
& \Phi_{2 \diamond 3}=\frac{1}{2}(1-y) \ln (1-y) \\
& \Phi_{4}=\frac{1}{2} \ln (1-x) \\
& \Phi_{5}=\frac{1}{2} \ln (1-y / x)
\end{aligned}
$$

Assuming the Standard Model V-A structure of the charged current the spectrum of the charged lepton vanishes at $x_{\ell}=1$ and the spectrum of the neutrino does not. The latter is also significantly harder, see solid lines in Fig. 3 a-b. The average (scaled) energy of the neutrino $\left\langle x_{\nu}\right\rangle$ is larger than $\left\langle x_{\ell}\right\rangle$ of the charged lepton. For the sake of simplicity we neglect QCD corrections and 
Figure 3. Energy distributions a) $\mathrm{A}_{1}\left(x_{\ell}\right)$ of the charged lepton and b) $\mathrm{A}_{\nu}\left(x_{\nu}\right)$ of the neutrino for the standard model V-A coupling $\left(\kappa^{2}=0\right)$ and an admixture of $\mathrm{V}+\mathrm{A}$ current $\left(\kappa^{2}=0.1\right)$ for $y=0.25$ and $\alpha_{s}=0.11$.

obtain the results shown in Table 2. It is interesting that for realistic range of $m_{t}$ the difference $\left\langle x_{\nu}-x_{\ell}\right\rangle$ is significantly greater than zero. This observation may be useful because a value close to zero can be expected for the background $b+W$ events of mass close to $m_{t}$ which do not come from $t$ decays and are not correlated by the dynamics. We give also the $\mathrm{V}-\mathrm{A}$ prediction for the average difference between $x_{>}=2 E_{>} / m_{t}$ of the more energetic and $x_{<}=2 E_{<} / m_{t}$ of the less energetic lepton. Let us remark that for hadronic decays, e.g. $t \rightarrow b W \rightarrow b \bar{d} u$, up-type quarks play the role of neutrinos and down-type antiquarks the role of charged leptons. Thus, $\left\langle x_{>}-x_{<}\right\rangle$ is also proportional to the average difference between the energies of the more and less energetic jets for hadronic decays of top quark. The values given in Table 2 are much larger than the result of a crude estimation assuming isotropic distribution of leptons in $W$ rest frame and statistically independent $W$ and $b$ which implies $\left\langle x_{>}-x_{<}\right\rangle_{b c k g}=\frac{1}{4}(1-y)$. However, the former assumption is not realistic in particular for $\bar{p} p$ colliders.
For $\mathrm{V}+\mathrm{A}$ coupling the charged lepton and the neutrino energy spectra would be interchanged in comparison to the $\mathrm{V}-\mathrm{A}$ case. In 23] effects have been studied of a small admixture of nonstandard $\mathrm{V}+\mathrm{A}$ current on distributions of leptons. The $t b W$ vertex has been parametrized as

$$
\begin{array}{r}
g_{V} \gamma^{\mu}+g_{A} \gamma^{\mu} \gamma_{5} \\
g_{V}=(1+\kappa) / \sqrt{1+\kappa^{2}} \\
g_{A}=(-1+\kappa) / \sqrt{1+\kappa^{2}}
\end{array}
$$

Hence $\kappa=0$ corresponds to pure V-A and $\kappa=\infty$ to $\mathrm{V}+\mathrm{A}$. In Fig. $3 \mathrm{a}-\mathrm{b}$ the lepton spectra are plotted corresponding to $\kappa^{2}=0.1$, see dashed lines. It can be seen that the deviations from the results of the Standard Model (solid lines) are rather small. They are larger for the polarization dependent distributions which are discussed in the following.

\subsection{Chirality of $b$ jet}

A measurement of $b$ quark chirality in top decay offers another opportunity to test the $\mathrm{V}$-A form of $t b W$ vertex. Chirality and helicity are nearly identical for the highly relativistic $b$ quark 
Table 2

Moments of the energy distributions of the leptons in $t \rightarrow b W \rightarrow b e^{+} \nu$ and the light quark jets in $t \rightarrow b W \rightarrow b \bar{d} u$ decays.

\begin{tabular}{|c|c|c|c|c|}
\hline & $\mathrm{y}$ & $m_{t}=150$ & $m_{t}=175$ & $m_{t}=200$ \\
\hline$\left\langle x_{\nu}\right\rangle$ & $\frac{1+4 y+y^{2}}{2(1+2 y)}$ & 0.707 & 0.663 & 0.631 \\
\hline$\left\langle x_{\ell}\right\rangle$ & $\frac{1+2 y+3 y^{2}}{2(1+2 y)}$ & 0.577 & 0.546 & 0.529 \\
\hline$\left\langle x_{\nu}-x_{\ell}\right\rangle$ & $\frac{y(1-y)}{1+2 y}$ & 0.130 & 0.117 & 0.102 \\
\hline$\left\langle x_{>}-x_{<}\right\rangle$ & $\frac{3\left(1+2 y-3 y^{2}\right)}{8(1+2 y)}$ & 0.317 & 0.340 & 0.353 \\
\hline
\end{tabular}

which originates from the decay, so, a high degree of primordial polarization is expected.

It has been proposed long ago [24] that distributions of charged leptons from semileptonic decays of beautiful hadrons can be used in polarization studies for $b$ quarks. Some advantages of neutrino distributions have been also pointed out [25,26]. Recently there has been considerable progress in the theory of the inclusive semileptonic decays of heavy flavor hadrons. It has been shown that in the leading order of an expansion in inverse powers of heavy quark mass $1 / m_{Q}$ the spectra for hadrons coincide with those for the decays of free heavy quarks [27] and there are no $\Lambda_{Q C D} / m_{Q}$ corrections to this result away from the energy endpoint. $\Lambda_{Q C D}^{2} / m_{Q}^{2}$ corrections have been calculated in 28,29 for $B$ mesons and in 29] for polarized $\Lambda_{b}$ baryons. For some decays the results are similar to those of the well-known $A C C M M$ model 30]. Perturbative first order QCD corrections to semileptonic decays of polarized $b$ quarks are also known 21,25,22, An old conflict between 31 and 21 can be considered as solved in favor of the results of the latter work.

The theory is therefore in good shape. The real drawback is that due to hadronization the net longitudinal polarization of the decaying $b$ quark is drastically decreased. In particular those $b$ quarks become depolarized which are bound in $B$ mesons both produced directly and from $B^{*} \rightarrow B \gamma$ transitions. Only those $b$ 's (a few percent) which fragment directly into $\Lambda_{b}$ baryons retain information on the original polarization 32,33. The signal is significantly reduced and feasibility of polarization studies for $b$ jets is problematic. The ongoing experimental studies of $b$ polarization at LEP should shed some light on this problem, see 34, 35 for a review. The reason is that $Z^{0} \rightarrow b \bar{b}$ decays can be viewed as a source of highly polarized $b$ quarks and the polarizations of $b$ jets in $Z^{0}$ and $t$ decays are similar. According to the Standard Model the polarization of $b$ quarks from $Z^{0}$ decays depends weakly on the production angle. The degree of longitudinal polarization is fairly large, amounting to $\left\langle P_{b}\right\rangle=-0.94$. QCD corrections to this Born result are about 3\% [36].

\subsection{Polarized Top Quarks}

The analysis of polarized top quarks and their decays has recently attracted considerable attention. Studies at a linear electron-positron collider are particularly clean for precision tests. However, also hadronic 37 39 or $\gamma \gamma$ collisions 40 and subsequent spin analysis of top quarks might reveal new information. These studies will result in determination of the top quark coupling to the $W$ and $Z$ bosons either confirming the predictions of the Standard Model or providing clues for physics beyond. The latter possibility is particularly intriguing for the top quark because $m_{t}$ plays an exceptional role in the fermion mass spectrum.

A number of mechanisms have been suggested that will lead to polarized top quarks. In 40 
this possibility has been discussed for $\gamma \gamma$ collisions with circular polarized photons. Related studies may be performed in hadronic collisions which in this case, however, are based on the correlation between $t$ and $\bar{t}$ decay products 38,41,39. The most efficient and flexible reactions producing polarized top quarks are electron-positron collisions. A small component of polarization transverse to the production plane is induced by final state interactions which have been calculated in perturbative QCD [37,39]. The longitudinal polarization $P_{L}$ is large. Its dependence on the production angle, beam energy and the top mass has been discussed in 41, 42]. $P_{L}$ varies strongly with the production angle, e.g. between nearly 0.6 for $\cos \vartheta=-1$ and -0.3 for $\cos \vartheta=1$ at $\sqrt{s}=500$ $\mathrm{GeV}$. Averaging over the production angle leads therefore to a significant reduction of $P_{L}$ with typical values of $\left\langle P_{L}\right\rangle$ around -0.2 [36]. QCD corrections change $\left\langle P_{L}\right\rangle$ by a relative amount of about $3 \%$ 36.

All these reactions lead to sizable polarization and can be used to obtain information on the production mechanism. However, two drawbacks are evident: production and decay are mixed in an intricate manner, and furthermore the degree of polarization is relatively small and depends on the production angle. Top quark production with longitudinally polarized electron beams and close to threshold provides one important exception: the restricted phase space leads to an amplitude which is dominantly S-wave such that the electron (and positron) spin is directly transferred to the top quark. Close to threshold and with longitudinally polarized electrons one may deal with a highly polarized sample of top quarks independent of the production dynamics. Thus one can study $t$ decays under particularly convenient conditions: large event rates, well identified restframe of the top quark, and large degree of polarization.

\subsubsection{Angular distributions}

In the rest frame of the decaying $t$ quark the angular distributions of the decay products are sensitive to its polarization. Let us define the angle $\theta_{\mathrm{w}}$ between $W$ boson three-momentum and the polarization three-vector $\vec{s}$. In the top quark rest frame $s=(0, \vec{s})$ is the polarization four-vector of the decaying top quark. Note that $S=|\vec{s}|=1$ corresponds to fully polarized and $S=0$ to unpolarized top quarks. We define also the angles $\theta_{+}$and $\theta_{0}$ between $\vec{s}$ and the directions of the charged lepton and the neutrino, respectively, and $\theta_{<}$for the less energetic lepton in semileptonic or less energetic light quark in hadronic decays. For the sake of simplicity let us confine our discussion to Born approximation and consider semileptonic $t \rightarrow b W \rightarrow b \ell^{+} \nu$ and hadronic $t \rightarrow b W \rightarrow b \bar{d} u$ decays.

The angular distribution of the charged lepton is of the form

$\frac{\mathrm{d} N}{\mathrm{~d} \cos \theta_{+}}=\frac{1}{2}\left[1+S \cos \theta_{+}\right]$

which follows, c.f. the following subsection, from factorization of the angular-energy distribution into an energy and an angular dependent part. This factorization holds for arbitrary top mass below and above the threshold for decays into real $W$ bosons 43.21]. It is noteworthy that for $S=1$ the angular dependence in (15) is maximal because a larger coeffecient multiplying $\cos \theta_{+}$ would be in conflict with positivity of the decay rate. Thus the polarization analysing power of the charged lepton angular distribution is maximal and hence far superior to other distributions discussed in the following. In particular the angular distribution of the neutrino reads [23]:

$\frac{\mathrm{d} N}{\mathrm{~d} \cos \theta_{0}}=\frac{1}{2}\left[1+h_{\nu}(y) S \cos \theta_{+}\right]$

where $h_{\nu}(y)$ is given in Table 3. The distribution of the direction of $W$ can be easily obtained. Only the amplitudes for the helicity states of $W$ $\lambda_{\mathrm{w}}=-1$ and $\lambda_{\mathrm{w}}=0$ are allowed and their contributions to the decay rate are in the ratio $2 y: 1$ [44. The corresponding angular distributions are of the form

$\frac{\mathrm{d} N_{-1,0}}{\mathrm{~d} \cos \theta_{\mathrm{w}}}=\frac{1}{2}\left(1 \mp S \cos \theta_{\mathrm{w}}\right)$

After summation over the $W$ polarizations the following angular dependence is obtained:

$\frac{\mathrm{d} N}{\mathrm{~d} \cos \theta_{\mathrm{w}}}=\frac{1}{2}\left[1+h_{\mathrm{w}}(y) S \cos \theta_{\mathrm{w}}\right]$ 
Table 3

Angular dependence of the distributions of $W$ bosons, neutrinos and less energetic leptons in $t \rightarrow b W \rightarrow$ $b e^{+} \nu$ or light quark jets in $t \rightarrow b W \rightarrow b \bar{d} u$ decays.

\begin{tabular}{ccccc}
\hline & $\mathrm{y}$ & $m_{t}=150$ & $m_{t}=175$ & $m_{t}=200$ \\
\hline$h_{\nu}(y)$ & $1-\frac{12 y(1-y+y \ln y)}{(1-y)^{2}(1+2 y)}$ & -0.521 & -0.311 & -0.127 \\
$h_{\mathrm{W}}(y)$ & $\frac{1-2 y}{1+2 y}$ & 0.275 & 0.410 & 0.515 \\
$h_{<}(y)$ & $1-\frac{6 y\{1-y-2 y \ln [(1+y) /(2 y)]\}}{(1-y)^{2}(1+2 y)}$ & 0.464 & 0.509 & 0.559 \\
\hline
\end{tabular}

where $h_{\mathrm{w}}(y)$ is also given in Table 3. It is evident that the charged lepton angular distribution is significantly more sensitive towards the polarization of $t$ than the angular distributions of $W$ and $\nu$. The charged lepton is likely to be the less energetic lepton because its energy spectrum is softer than that of the neutrino. For large values of $m_{t}$ the angular distribution of the less energetic lepton

$\frac{\mathrm{d} N}{\mathrm{~d} \cos \theta_{<}}=\frac{1}{2}\left[1+h_{<}(y) S \cos \theta_{<}\right]$

is a more efficient analyser of top polarization than the angular distribution of neutrinos. For $m_{t}$ in the range $150-200 \mathrm{GeV}$ it is also better than the direction of $W$, c.f. Table 3 .

\subsubsection{Angular-energy distributions}

For semileptonic decays the normalized distributions of leptons including first order QCD corrections can be cast into the following form:

$\frac{\mathrm{d} N}{\mathrm{~d} x_{\ell} \mathrm{d} \cos \theta_{+}}=\frac{1}{2}\left[\mathrm{~A}_{\mathrm{l}}\left(x_{\ell}\right)+S \cos \theta_{+} \mathrm{B}_{\mathrm{l}}\left(x_{\ell}\right)\right]$

$\frac{\mathrm{d} N}{\mathrm{~d} x_{\nu} \mathrm{d} \cos \theta_{0}}=\frac{1}{2}\left[\mathrm{~A}_{\nu}\left(x_{\nu}\right)+S \cos \theta_{0} \mathrm{~B}_{\nu}\left(x_{\nu}\right)\right]$

where the functions $\mathrm{A}_{1}(x)$ and $\mathrm{A}_{\nu}(x)$ have been defined in eqs. (7) and (8), and

$$
\begin{aligned}
\mathrm{B}_{1}(x) & =12 \frac{\mathrm{J}_{0}^{+}(x, y)-a_{s} \mathrm{~J}_{1}^{+}(x, y)}{\mathcal{F}_{0}(y)-a_{s} \mathcal{F}_{1}(y)} \\
\mathrm{B}_{\nu}(x) & =12 \frac{\mathrm{J}_{0}^{-}(x, y)-a_{s} \mathrm{~J}_{1}^{-}(x, y)}{\mathcal{F}_{0}(y)-a_{s} \mathcal{F}_{1}(y)}
\end{aligned}
$$

where

$$
\begin{aligned}
& \mathrm{J}_{0}^{+}(x, y)=\mathrm{F}_{0}^{+}(x, y) \\
& \mathrm{J}_{0}^{-}(x, y)=(x-y)(1-x+y-2 y / x)
\end{aligned}
$$

and 45,22

$$
\begin{aligned}
& \mathrm{J}_{1}^{+}(x, y)=\mathrm{J}_{0}^{+}(x, y) \Phi_{0}-x \Phi_{1}+(5-2 x-y \\
& \quad-2 y / x-2 / x) \Phi_{2 \diamond 3}+(-3+x+2 / x) \Phi_{4} \\
& \quad+\left(-2 x y+3 x-4 x^{2}+6 y-y^{2}-2 y^{2} / x\right) \Phi_{5} \\
& \quad+\left(2-2 x^{2}+2 y-3 y^{2}-2 y / x+3 y^{2} / x\right) / 2
\end{aligned}
$$

$$
\begin{gathered}
\mathrm{J}_{1}^{-}(x, y)=\mathrm{J}_{0}^{-}(x, y) \Phi_{0}+(-2 x y-x-5 y+ \\
\left.y^{2}-2 y^{2} / x\right) \Phi_{1}+(3+10 x+y+10 y / x \\
-2 / x) \Phi_{2 \diamond 3}+\left(-3+12 x y+x-7 y-y^{2}\right. \\
\left.-12 y / x+8 y^{2} / x+2 / x\right) \Phi_{4}+(6 x y-9 x \\
\left.-4 x^{2}-y-2 y^{2}+10 y^{2} / x\right) \Phi_{5}+(2-5 x y \\
\left.-2 x^{2}+2 y+7 y^{2}-2 y / x-2 y^{2} / x\right) / 2
\end{gathered}
$$

Eq.(24) implies that in Born approximation the double differential angular-energy distribution of the charged lepton is the product of the energy distribution and the angular distribution (15). QCD corrections essentially do not spoil this factorization 445. For the neutrino such factorization does not hold, c.f. eqs.(10) and (25). After integration over $x_{\nu}$ the angular dependence of the neutrino distribution is much weaker than for the charged lepton, c.f. eq.(16).

In Fig. 19 the functions $\mathrm{B}_{1}(x)$ and $\mathrm{B}_{\nu}(x)$ are shown as solid lines for $y=0.25$ and $\alpha_{s}=0.11$ [23]. The effect of non-standard coupling defined in eq. (14) is much stronger for the polarization dependent 
Figure 4. Angular-energy distribution functions in the Standard Model $\left(\kappa^{2}=0\right)$ and for the admixture of $\mathrm{V}+\mathrm{A}$ current $\left(\kappa^{2}=0.1\right)$ : a) $\mathrm{B}_{1}\left(x_{\ell}\right)$ for the charged lepton and b) $\mathrm{B}_{\nu}\left(x_{\nu}\right)$ for the neutrino, $y=0.25$ and $\alpha_{s}=0.11$.

distribution of neutrinos, see dashed lines in Fig. 4 corresponding to $\kappa^{2}=0.1$ [23]. In Table 1 the moments

$$
\begin{aligned}
\mathcal{A}_{k}^{(\ell, \nu)} & =\int_{y}^{1} \mathrm{~d} y x^{k} \mathrm{~A}_{\ell, \nu}(x, y) \\
\mathcal{B}_{k}^{(\ell, \nu)} & =\int_{y}^{1} \mathrm{~d} y x^{k} \mathrm{~B}_{\ell, \nu}(x, y)
\end{aligned}
$$

are given for integer $k$ between -1 and $3, y=0.25$ and $\alpha_{s}\left(m_{t}\right)=0.11$ [23]. The upper entries in the table denote the values of the moments for $\kappa^{2}=0$ and the lower ones the ratios of the moments evaluated for $\kappa^{2}=0.1$ to those for $\kappa^{2}=0$. It is evident that the moments $\mathcal{B}_{k}^{(\nu)}$ which govern the angular dependence of the neutrino spectrum are particularly sensitive towards a $\mathrm{V}+\mathrm{A}$ admixture. The effect is most pronounced for the moment $k=-1$ which enhaces the lower energy part of the spectrum and where the relative change amounts to $17 \%$ for $\kappa^{2}=0.1$. Thus, the angular-energy distribution of neutrinos from the polarised top quark decay will allow for a particularly sensitive test of the V-A structure of the charged current. The effect of QCD correction can mimic a small admixture of $\mathrm{V}+\mathrm{A}$ interaction. Therefore, inclusion of the radiative QCD correction to the decay distributions is necessary for a quantitative study.

\section{TOP QUARK PAIR PRODUCTION}

3.1. $\mathrm{e}^{+} \mathrm{e}^{-} \rightarrow \mathrm{t} \overline{\mathrm{t}}$

It is evident that the bulk of top studies at an $e^{+} e^{-}$collider will rely on quarks produced in $e^{+} e^{-}$annihilation through the virtual $\gamma$ and $\mathrm{Z}$, with a production cross section of the order of $\sigma_{\text {point }} t^{2}$. For quarks tagged at an angle $\vartheta$, the differential cross section in Born approximation is a binomial in $\cos \vartheta$

$$
\begin{aligned}
\frac{d \sigma}{d \cos \vartheta}= & \frac{3}{8}\left(1+\cos ^{2} \vartheta\right) \sigma_{U}+\frac{3}{4} \sin ^{2} \vartheta \sigma_{L} \\
& +\frac{3}{4} \cos \vartheta \sigma_{F}
\end{aligned}
$$

$U$ and $L$ denote the contributions of unpolarized and longitudinally polarized gauge bosons along the $\vartheta$ axis, and $F$ denotes the difference between

\footnotetext{
${ }^{2}$ This subsection is a shortened version of a comprehensive review given in 12 .
} 
Table 4

The moments $\mathcal{A}_{k}^{(\ell, \nu)}$ and $\mathcal{B}_{k}^{(\ell, \nu)}$ of the angular-energy distributions for the charged leptons and for the neutrinos for $y=0.25$ and $\alpha_{s}=0.11$ for $\kappa^{2}=0$ (upper entries) and the ratios between the moments for $\kappa^{2}=0.1$ and $\kappa^{2}=0$ (lower entries).

\begin{tabular}{rrrrr}
\hline & $\mathcal{A}_{k}^{(\ell)}$ & $\mathcal{B}_{k}^{(\ell)}$ & $\mathcal{A}_{k}^{(\nu)}$ & $\mathcal{B}_{k}^{(\nu)}$ \\
\hline-1 & 2.008 & 2.005 & 1.593 & -0.707 \\
& .981 & .940 & 1.023 & 1.166 \\
& & & & -0.452 \\
0 & 1.000 & .998 & 1.000 & 1.110 \\
& 1.000 & .949 & 1.000 & -0.322 \\
& .559 & .558 & & 1.068 \\
& 1.021 & .960 & .683 & -0.249 \\
& .345 & .344 & .984 & 1.037 \\
& 1.043 & .973 & .500 & -0.203 \\
& .230 & .230 & .973 & 1.015 \\
\hline
\end{tabular}

right and left polarizations. The total cross section is the sum of $U$ and $L$ :

$\sigma=\sigma_{U}+\sigma_{L}$

In Born approximation the coefficients $\sigma^{i}$ can be expressed in terms of the cross sections for the massless case

$$
\begin{aligned}
\sigma_{B}^{U} & =\beta \sigma^{V V}+\beta^{3} \sigma^{A A} \\
\sigma_{B}^{L} & =\frac{1}{2}\left(1-\beta^{2}\right) \beta \sigma^{V V} \\
\sigma_{B}^{F} & =\beta^{2} \sigma^{V A}
\end{aligned}
$$

where

$$
\begin{aligned}
\beta= & \sqrt{1-4 m_{t}^{2} / s} \\
\sigma^{V V} & =\frac{4 \pi \alpha^{2}(s) e_{e}^{2} e_{Q}^{2}}{s} \\
& +\frac{G_{F} \alpha(s)}{\sqrt{2}} e_{e} e_{Q}\left(v_{e}+\rho a_{e}\right) v_{Q} \frac{m_{Z}^{2}\left(s-m_{Z}^{2}\right)}{D\left(s, m_{Z}, \Gamma_{Z}\right)} \\
& +\frac{G_{F}^{2}}{32 \pi}\left(v_{e}^{2}+a_{e}^{2}+2 \rho v_{e} a_{e}\right) v_{Q}^{2} \frac{m_{Z}^{4} s}{D\left(s, m_{Z}, \Gamma_{Z}\right)} \\
\sigma^{A A} & =\frac{G_{F}^{2}}{32 \pi}\left(v_{e}^{2}+a_{e}^{2}+2 \rho v_{e} a_{e}\right) a_{Q}^{2} \frac{m_{Z}^{4} s}{D\left(s, m_{Z}, \Gamma_{Z}\right)}
\end{aligned}
$$

$$
\begin{aligned}
\sigma^{V A} & =\frac{G_{F} \alpha(s)}{\sqrt{2}} e_{e}\left(\rho v_{e}+a_{e}\right) e_{Q} a_{Q} \frac{m_{Z}^{2}\left(s-m_{Z}^{2}\right)}{D\left(s, m_{Z}, \Gamma_{Z}\right)} \\
& +\frac{G_{F}^{2}}{16 \pi}\left[2 v_{e} a_{e}+\rho\left(v_{e}^{2}+a_{e}^{2}\right)\right] \frac{v_{Q} a_{Q} m_{Z}^{4} s}{D\left(s, m_{Z}, \Gamma_{Z}\right)}
\end{aligned}
$$

and

$$
D\left(s, m_{Z}, \Gamma_{Z}\right)=\left(s-m_{Z}^{2}\right)^{2}+\left(s \Gamma_{Z} / m_{Z}\right)^{2}
$$

The fermion couplings are given by

$$
v_{F}=2 I_{3}^{f}-4 e_{f} \sin ^{2} \theta_{w} \quad, \quad a_{f}=2 I_{3}^{f}
$$

and the possibility of longitudinal electron polarization $(\rho=-1 ;+1 ; 0$ for righthanded; lefthanded; unpolarized electrons) has been included. QCD corrections to this formula are available for arbitrary $\mathrm{m}^{2} / \mathrm{s}$ up to first order in $\alpha_{s}$ :

$\sigma=\frac{\left(3-\beta^{2}\right)}{2} \beta \sigma^{V V} R_{V}+\beta^{3} \sigma^{A A} R_{A}$

The exact result [46] for the QCD enhancement factors can be well approximated by [4]

$R_{V}=1+\frac{4 \alpha_{s}}{3}\left[\frac{\pi}{2 \beta}-\frac{3+\beta}{4}\left(\frac{\pi}{2}-\frac{3}{4 \pi}\right)\right]$ 
Figure 5. Cross section for $t \bar{t}$ production in units of $\sigma_{\text {point }}$ including resonance and QCD enhancement and initial state radiation.

and

$$
\begin{aligned}
& R_{A}= \\
& 1+\frac{4 \alpha_{s}}{3}\left[\frac{\pi}{2 \beta}-\left(\frac{19}{10}-\frac{22}{5} \beta+\frac{7}{2} \beta^{2}\right)\left(\frac{\pi}{2}-\frac{3}{4 \pi}\right)\right]
\end{aligned}
$$

For small $\beta$ these factors develop the familiar Couloumb enhancement $\sim \frac{2}{3} \pi \alpha_{s} / \beta$ compensating the phase space suppression $\sim \beta$. This leads to a nonvanishing cross section which smoothly joins the threshold region.

Initial state radiation has an important influence on the magnitude of the cross section which is significantly suppressed in the threshold region. The correction factor increases rapidly with energy, but stays below 0.9 in the full range under consideration. In Fig. 5 the cross section for $e^{+} e^{-} \rightarrow t \bar{t}$ is shown as the function of the centerof-mass energy [12].

\subsection{Pair production near energy threshold}

It follows from Table 1 that the top quark is a short-lived particle. Its width $\Gamma_{t}$ is in the range $1-2 \mathrm{GeV}$ and fairly exceeds the tiny hyperfine splitting for open top hadrons, the hadronization scale of about $200 \mathrm{MeV}$, and even the energy splitting between $1 S$ and $2 S t \bar{t}$ resonances. On one side this is an advantage because long-distance phenomena related to confinement are apparently less important for top quarks [48]. In particular depolarization due to hadronization is practically absent. On the other side the amount of information available from the threshold region is significantly reduced. Toponium resonances including $1 S$ overlap each other. As a consequence the cross section for $t \bar{t}$ pair production near energy threshold has a rather simple and smooth shape. Nevertheless, as first pointed out by Fadin and Khoze [49] the excitation curve $\sigma\left(e^{+} e^{-} \rightarrow t \bar{t}\right)$ allows a precise determination of $m_{t}$ as well as of other physical quantities such as $\Gamma_{t}$ and the strong coupling constant. The results of 49] for the Coulomb chromostatic potential have been derived analytically in the non-relativistic approximation. Strassler and Peskin [50] have obtained similar results using numerical approach and a more realistic QCD potential. The idea 449,50] to use Green function instead of summing over overlapping resonances has been also applied in calculations of differential cross sections 51,55 . Independent numerical approaches have been devoleped for solving Schrödinger equation in the position space [51] and Lippmann-Schwinger equation in the momentum space [52,53]. The results of these two methods agree very well [54]. One of the most important future applications will be in determination of $m_{t}$ and $\alpha_{s}$. It has been argued [51] and demonstrated [54,55] that the combined measurements of the total and the differential cross sections in $e^{+} e^{-} \rightarrow t \bar{t}$ offer a very 
Figure 6. Distribution $|p \mathcal{G}(\vec{p}, E)|^{2}$ of the momentum $p=|\vec{p}|$ of the top quark in $t \bar{t}$ system for $E=-2.9 \mathrm{GeV}$ (1S peak), $E=0$ and $E=2 \mathrm{GeV}$ a) $m_{t}=150$ and b) $180 \mathrm{GeV}$.

promising method for a simultaneous determination of $m_{t}$ and $\alpha_{s}\left(m_{t}\right)$. The estimated errors of such a determination are [54]: $\Delta m_{t}=300 \mathrm{MeV}$ and $\Delta \alpha_{s}=0.006$ for $m_{t}=150 \mathrm{GeV}$ and $\Delta m_{t}=520$ $\mathrm{MeV}$ and $\Delta \alpha_{s}=0.009$ for $m_{t}=180 \mathrm{GeV}$. The errors are correlated, so for $\alpha_{s}$ fixed by other measurements a much better determination of $m_{t}$ can be obtained. In the following the method of [52,53] is presented. Some theoretical uncertainties related to the momentum dependent width of $t \bar{t}$ system, long-distance part of the QCD potential and the definition of the pole top quark mass are discussed. Effects of Higgs exchange are also considered.

\subsubsection{Green function}

Let us describe briefly the Green function method for $e^{+} e^{-} \rightarrow t \bar{t}$ annihilation near the energy threshold and the numerical solution of the Lippmann-Schwinger equation; see 52,53 for details. We simplify the original calculations and neglect the $Z^{0}$ contribution and the transverse gluon correction to the production vertex. The differential cross section for the top quark pair production in electron-positron annihilation reads:

$$
\frac{\mathrm{d} \sigma}{\mathrm{d}^{3} p}(\vec{p}, E)=\frac{3 \alpha^{2} Q_{t}^{2}}{\pi s m_{t}^{2}} \Gamma(p, E)|\mathcal{G}(\vec{p}, E)|^{2}
$$

where $\Gamma(p, E)=\Gamma_{t \bar{t}} / 2$. The Green function $\mathcal{G}(\vec{p}, E)$ is the solution of the non-relativistic Lippmann-Schwinger equation

$$
\begin{aligned}
& \mathcal{G}(\vec{p}, E)=\mathcal{G}_{0}(\vec{p}, E)+ \\
& \mathcal{G}_{0}(\vec{p}, E) \int \frac{\mathrm{d}^{3} q}{(2 \pi)^{3}} \tilde{V}(\vec{p}-\vec{q}) \mathcal{G}(\vec{q}, E)
\end{aligned}
$$

where

$E=\sqrt{s}-2 m_{t}$

denotes the total energy of the $t \bar{t}$ system and $\vec{p}$ the momentum of $t$ quark. $\tilde{V}(\vec{p})$ is the potential in momentum space and $\Gamma(p, E)=\Gamma_{t \bar{t}} / 2$, where $\Gamma_{t \bar{t}}$ denotes the width of the the $t \bar{t}$ system. The free Hamiltonian that is used to define the Green function $\mathcal{G}_{0}$ includes the momentum dependent width:

$\mathcal{G}_{0}(\vec{p}, E)=\frac{1}{E-\frac{p^{2}}{m_{t}}+\mathrm{i} \Gamma(p, E)}$

Near the energy threshold one can neglect all but $S$ partial waves and solve numerically the corresponding one-dimensional integral equation. The spherically symmetric solution fulfills the unitarity condition [5]

$$
\begin{aligned}
\int \frac{\mathrm{d}^{3} p}{(2 \pi)^{3}} \Gamma(p, E)|\mathcal{G}(p, E)|^{2}= \\
-\operatorname{Im} G\left(\vec{x}=0, \vec{x}^{\prime}=0, E\right)
\end{aligned}
$$


which for the constant decay rate reduces to the formula for the total cross section derived in 49,50. Eq. (45) can be also considered as a nontrivial cross check of the numerical approach. In Fig.6 the momentum distributions of $t$ quarks in $t \bar{t}$ systems of energy $E$ corresponding to $1 S$ peak , $E=0$ and $E=2 \mathrm{GeV}$ are shown as solid, dotted and dashed lines respectively. The shift towards larger momenta with increasing $E$ and the narrowing of the distributions is clearly visible. It has been shown [54,55 that the position of the maximum is not sensitive to the initial state radiation, so it can be used to measure $E$ and $m_{t}$. On the other hand the rapid increase of the cross section $\sigma\left(e^{+} e^{-} \rightarrow t \bar{t}\right)$ is related to the location of $1 S$ resonance which depends on both $m_{t}$ and $\alpha_{s}$. Thus, the combined measurement of both the total and differential cross sections leads to a simultaneous determination of these parameters. The results shown in Fig. 6 have been obtained in [52 assuming constant width of $t \bar{t}$ system $\Gamma(p, E)=\Gamma_{t}$. Let us discuss now theoretical problems which appear when this assumption is lifted.

\subsubsection{Width of $t \bar{t}$ system.}

The width of the $t \bar{t}$ system depends on the momentum of $t$ quark because both the matrix element and the phase space available for the decay products depend on it. When produced near energy threshold $t$ and $\bar{t}$ cannot be considered as free particles. The binding energy and the kinetic energy of internal motion tend to reduce the available phase space for the decay. Although the effect is only $\mathcal{O}\left(\alpha_{s}{ }^{2}\right)$ the suppression can be significant [56]. Thus, in a high precision calculation one has to consider the width $\Gamma_{t-\bar{t}}(p)$ as a non-trivial function of the momentum $p$. The phase space effect tends to reduce the decay rate of bound top quarks relative to free ones [56,51] and the effect is enhanced because for short-lived particles the momentum distribution is broad. However, for the same reason the decays take place at short relative distances, where the wave functions of $b$ and $\bar{b}$ quarks originating from the decays are distorted (enhanced) by Coulomb attraction. Therefore, when calculating the amplitude of $t \rightarrow b W$ transition, one should
Figure 7. Comparison of the annihilation cross section $\sigma\left(e^{+} e^{-} \rightarrow t \bar{t}\right)$ evaluated for the constant width $\Gamma_{t}$ (dashed line) and the momentum dependent widths including time dilatation (solid line) and phase space suppression (dotted line).

use Coulomb wave functions rather than plane waves for $b$ quarks. This effect clearly increases the rate. A third factor is due to time dilatation: a top quark moving with velocity $v$ lives longer in the center-of-mass frame. While phase space reduction and time dilatation can be implemented in a straightforward way Coulomb enhancement cannot be easily taken into account. In principle one has to replace the plane wave functions for $b$ quarks by relativistic Coulomb functions when evaluating the amplitude for the $t \rightarrow b W$ transition. In Fig.7 [53 predictions for the annihilation cross section $\sigma\left(e^{+} e^{-} \rightarrow t \bar{t}\right)$ are shown corresponding to different asumptions about $\Gamma_{t \bar{t}}$ : solid line has been obtained including only time dilatation, dotted line including only the phase space suppression and the dashed line corresponds to the constant width. The effect of the phase space suppression is non-negligible whereas time dilatation produces rather small effect.

It has been conjectured [52,53,58 that, in close analogy to what happens in the case of negative muons bound in nuclei [57], for chromostatic attraction in $t \bar{t}$ systems the phase space suppression and the Coulomb enhancement cancel each other and only the suppression due to time dilatation should be included. Recently this conjecture has 
been proven [59]. Moreover, it has been explicitly demonstrated that unphysical gauge dependence of the momentum dependent width [51] also disappeares in the final result [59. The distributions of the top quark momentum, see Fig.6, are also only weakly affected. It should be stressed, however, that the experimentally accessible threemomentum distributions of $W b$ systems from top quark decays are not identical to the distributions of the top quark momentum. Calculable corrections arise due to final state rescattering [55].

\subsubsection{QCD potential}

Figure 8. $\alpha_{\text {eff }}(q)$ for different values of $\alpha_{s}\left(m_{\mathrm{z}}\right)$ : solid -0.12 , dashed -0.11 , dash-dotted -0.13 and dotted -0.10 and 0.14 .

Some difficulties appear also for the QCD potential. In order to illustrate these problems let us consider the following potential [53] defined in momentum space $(p=|\vec{p}|)$ :

$\tilde{V}_{J K T}(p)=-\frac{16 \pi}{3} \frac{\alpha_{e f f}(p)}{p^{2}}+V_{0} \delta(p)$

The function

$\alpha_{e f f}(p)= \begin{cases}\alpha_{\text {pert }}(p) & \text { if } p>p_{1} \\ \alpha_{R}(p) & \text { if } p<p_{2}\end{cases}$
Figure 9. QCD potential $V_{J K T}(r)$ for different values of $\alpha_{s}\left(m_{\mathrm{z}}\right)$ : solid -0.12 , dashed -0.11 , dash-dotted -0.13 and dotted -0.10 and 0.14 .

is shown in Fig.8. At large momenta, i.e. for $p>p_{1}=5 \mathrm{GeV} V_{J K T}(p)$ is equal to the perturbative two-loop QCD potential 60 for $n_{f}=5$ quark flavours and $\alpha_{s}\left(m_{\mathrm{z}}\right)=0.10-0.14$. At intermediate and small momenta, i.e. for $p<p_{2}=2$ $\mathrm{GeV}$ a Richardson-like phenomenological potential 61] is employed, and a linear interpolation formula is used in between. While the form of the potential in momentum space is fixed at large and intermediate momenta by perturbative QCD and phenomenology of $b \bar{b}$ and $c \bar{c}$ quarkonia, it is not known in the infrared (confinement) region. Thus $V_{0}$ in eq.(46) is to some extent an arbitrary parameter. In 56] $V_{0}$ has been fixed by imposing an evidently arbitrary condition on the potential in the position space

$V_{J K T}\left(r=1 \mathrm{GeV}^{-1}\right)=-1 / 4 \mathrm{GeV}$

Then after Fourier transformation the potential $V_{J K T}(r)$ is obtained, see Fig.9, which describes well the $b \bar{b}$ and $c \bar{c}$ quarkonia. A different choice of $V_{0}$ results in a redefinition of $b$ and $c$ quark masses which in the framework of the non-relativistic approach are defined only with limited $(\sim 300 \mathrm{MeV})$ precision. In particular one can impose conditions relating $V_{0}$ and $\alpha_{s}\left(m_{\mathrm{z}}\right)$ which lead to different energy dependence of the momentum distributions 
in $t \bar{t}$ systems. At this point a brief discussion is in order on the relation between the top masses determined on the basis of different potentials [62. The perturbative two-loop QCD potential in momentum space is fixed unambiguously for sufficiently large $Q^{2}$. In order to calculate the potential in coordinate space the small $Q^{2}$ behavior has to be specified in an ad hoc manner. Different assumptions will lead to the same short distance behavior. The potentials will, however, differ with respect to their long distance behavior. In 49 it has been argued convincingly that the long distance tail is cut off by the large top width. However, an additive constant in coordinate space can be induced by the small momentum part of $\tilde{V}(p)$. This additional term leads to a shift in the $t \bar{t}$ threshold, which in turn can be reabsorbed by a corresponding shift in $m_{t}$. The different assumptions on the long distance behavior are reflected in differences between the predictions of 50,51.53. for the precise location of the $t \bar{t}$ threshold for identical values of $\alpha_{s}$ and $m_{t}$ as well as in differences in the $\alpha_{s}$ dependence of the momentum distributions for fixed $m_{t}$ and energy, see also [54. All these differences can be attributed to the freedom in the additive constant discussed before. The same additive constant appears in $b \bar{b}$ spectroscopy, such that the mass difference between top and bottom is independent from these considerations. Recently problems with the definion of $b$ quark mass due to long distance effects (infrared renormalons) have been noted in [63].

A similar study has been performed in 64]. In Fig $10 \sigma\left(e^{+} e^{-} \rightarrow t \bar{t}\right)$ is shown as the function of energy relative to the location of the $1 S$ peak $\Delta E=\sqrt{s}-E_{1 s}$. The solid lines have been obtained for the potential of [51] and the dashed ones for the potential of [50]. These two potentials are equal at short distances but different prescriptions are used at intermediate and large distances. It can be seen that the height of the $1 S$ peak is affected. The ambiguity may be fixed by performing a simultaneous fit to the differential momentum distribution, angular distributions (e.g. the forward-backward asymmetries) and/or by fixing $\alpha_{s}$ from independent measurements. It is clear that this problem deserves a careful further study.
Figure 10. Comparison of $t \bar{t}$ cross section as the function of energy relative to the position of $1 S$ peak for different potentials 64 .

\subsubsection{Higgs effects in the threshold region} Effects of Higgs exchange may be nonnegligible in the threshold region [50] in particular if the Higgs mass is relatively low as predicted by supersymetric models. The dominant effect from a light Higgs boson can be described by an instantaneous Yukawa potential

$V_{Y u k}(r)=-\kappa \frac{e^{-m_{h} r}}{r}$

with

$\kappa=\sqrt{2} G_{F} m_{t}^{2} / 4 \pi$

The vertex correction from Higgs exchange can be included in the potential calculation by adding $V_{Y u k}$ to the QCD potential:

$V_{e f f}=V_{Q C D}+V_{Y u k}$

Then, $V_{\text {eff }}$ can be used instead of $V_{Q C D}$ in numerical calculations of the total [50] and differential [51,52] cross sections for $e^{+} e^{-}$annihilation near $t \bar{t}$ threshold. The dominant contribution to the remainder can be included as an overall correction factor to the amplitude 65:

$1+\frac{\kappa}{\pi}\left[f_{t h r}\left(m_{h}^{2} / m_{t}^{2}\right)-\pi \frac{m_{t}}{m_{h}}\right]$ 
where 66 68

$$
\begin{array}{r}
f_{t h r}(r)=-\frac{1}{12}\left[-12+4 r+\left(-12+9 r-2 r^{2}\right) \ln r\right. \\
\left.+\frac{2}{r}\left(-6+5 r-2 r^{2}\right) l_{4}(r)\right] \\
l_{4}(r)= \begin{cases}\sqrt{r(4-r)} \arccos (\sqrt{r} / 2) & \text { if } r \leq 4 \\
-\sqrt{r(r-4)} \frac{1}{2} \ln \frac{1+\sqrt{1-4 / r}}{1-\sqrt{1-4 / r}} & \text { if } r>4\end{cases}
\end{array}
$$

This factor is energy independent and can be used below as well as above the threshold.

\section{Acknowledgements}

I would like to gratefully acknowledge the collaboration with A. Czarnecki, C. Jünger, J.H. Kühn and T. Teubner on many aspects of top quark physics covered in this review. I thank I. Bigi, E. Boos, A. Buras, K. Hagiwara, P. Igo-Kemenes, V. Khoze, M. Martinez, R. Miquel, M. Peskin, H. Pilkuhn, M.-L. Stong, Y. Sumino, M. Veltman, K. Zalewski and P. Zerwas for useful comments and discussions.

\section{REFERENCES}

1. S. Abachi et al. (D0 Collaboration), preprint FERMILAB-PUB-94/004-E.

2. A. Blondel, in these proceedings.

3. F. Abe et al. (CDF Collaboration), preprint FERMILAB-PUB-94/097-E.

4. M. Raidal and R. Vuopionperä, Phys. Lett. B318 (1993) 237.

5. E. Boos et al., Phys. Lett. B326 (1994) 190.

6. S.S.D. Willenbrock and D.A. Dicus, Phys. Rev. D34 (1986) 155;

C.P. Yuan, Phys. Rev. D41 (1990) 42;

G.V. Jikia and S.R. Slabospitsky, Phys. Lett. B295 (1992) 136.

7. R. Orava, P. Eerola and M. Nordberg (eds.), Physics and Experiments with Linear Colliders, World Scientific, 1992.

8. F.A. Harris, S.L. Olsen, S. Pakvasa and X. Tata, Physics and Experiments with Linear $e^{+} e^{-}$Colliders, World Scientific, 1993.
9. P.M. Zerwas (ed.), $e^{+} e^{-}$Collisions at 500 GeV: The Physics Potential, DESY Orange Reports DESY 92-123A, DESY 92-123B and DESY 93-123C.

10. J.H. Kühn and P.M. Zerwas, in A.J. Buras and M. Lindner (eds.), Heavy Flavours in High Energy Electron-Positron Collisions, World Scientific, 1992.

11. P.M. Zerwas, in ref. [7], vol.I, p.165.

12. J.H. Kühn (conv.) et al., in ref.[9], Part A, p. 255 .

13. J.H. Kühn, in ref.[8], vol.I, p.72.

14. P. Igo-Kemenes (conv.) et al., in ref.|9], Part A, p.327.

15. M. Aguilar-Benitez et al., Phys. Rev. D45 (1992) Part 2.

16. M. Jeżabek and J.H. Kühn, Nucl. Phys. B314 (1989) 1.

17. A. Denner and T. Sack, Nucl. Phys. B358 (1991) 46; G.Eilam, R.R.Mendel, R.Migneron and A.Soni, Phys. Rev. Lett. 66 (1991) 3105.

18. B.A. Irwin, B. Margolis and H.D. Trotier, Phys. Lett. B256 (1991) 533.

19. M. Jeżabek and J.H. Kühn, Phys. Rev. D48 (1993) R1910.

20. A. Czarnecki, M. Jeżabek and J.H. Kühn, Acta Phys. Polonica B20 (1989) 961, (E) B23 (1992) 173; M. Jeżabek and C. Jünger, Acta Phys. Polonica B24 (1993) 1923.

21. M. Jeżabek and J.H. Kühn, Nucl. Phys. B320 (1989) 20.

22. A. Czarnecki and M. Jeżabek, preprint TTP 93-40, Karlsruhe, 1994, hep-ph/9402326, Nucl. Phys. B, in print.

23. M. Jeżabek and J.H. Kühn, preprint TTP 94-4, Karlsruhe, 1994, hep-ph/9403366; Phys.Lett. B (1994) in print.

24. G. Köpp, L.M. Sehgal and P.M. Zerwas, Nucl. Phys. B123 (1977) 77;

B. Mele and G. Altarelli, Phys. Lett. B299 (1993) 345 .

25. A. Czarnecki, M. Jeżabek, J.G. Körner and J.H. Kühn, preprint TTP 93-32, Karlsruhe, 1993, Phys. Rev. Lett., in print.

26. G. Bonvicini and L. Randall, preprint CERNPPE/94-07, 1994.

27. J. Chay, H. Georgi and B. Grinstein, Phys. Lett. B247 (1990) 399. 
28. I. Bigi, M. Shifman, N. Uraltsev and A. Vainshtein, Phys.Rev.Lett. 71 (1993) 496.

29. A.V. Manohar and M.B. Wise, Phys. Rev. D49 (1994) 1310.

30. Altarelli, G. et al, Nucl. Phys. B208 (1982) 365 .

31. N. Cabibbo, G. Corbo and L. Maiani, Nucl. Phys. B155 (1979) 93;

G. Corbo, Nucl. Phys. B212 (1983) 99.

32. J.D. Bjorken, Phys. Rev. D40 (1989) 1513.

33. F.E. Close, J.G. Körner, R.J.N. Phillips and D.J. Summers, J. Phys. G18 (1992) 1716.

34. J.H. Kühn, P.M. Zerwas et al., in G. Altarelli et al. (eds.), Z Physics at LEP I, CERN Report CERN 89-08, vol.I, p.267.

35. B. Mele, preprint n.1009, Rome, 1994.

36. J.G. Körner, A. Pilaftsis and M.M. Tung, preprint MZ-TH/93-30.

37. A. Devoto, J. Pumplin, W. Repko and G.L. Kane, Phys. Rev. Lett. 43 (1979) 105.

38. J.H. Kühn, Nucl. Phys. B237 (1984) 77.

39. G.L. Kane, G.A. Ladinsky and C.-P. Yuan, Phys.Rev. D45 (1992) 124; T. Arens and L.M. Sehgal, Phys. Lett. B302 (1993) 501.

40. V.S. Fadin, V.A. Khoze and M.I. Kotsky, preprint hep-ph/9403246.

41. J.H. Kühn, A. Reiter and P.M. Zerwas, Nucl. Phys. B272 (1986) 560.

42. R.H. Dalitz and G.R. Goldstein, Phys. Rev. D45 (1992) 1531;

T. Arens and L.M. Sehgal, Nucl. Phys. B393 (1993) 46.

43. J.H. Kühn, Acta Phys. Austriaca, Suppl. 24 (1982) 71; J.H. Kühn and K.H. Streng, Nucl. Phys. B198 (1982) 71.

44. F.J. Gilman and R. Kauffman, Phys. Rev. D37 (1988) 2678.

45. A. Czarnecki, M. Jeżabek and J.H. Kühn, Nucl. Phys. B351 (1991) 70.

46. J. Jersak, E. Laermann and P.M. Zerwas, Phys. Rev. D25 (1982) 363;

J. Schwinger, Particles, Sources and Fields, Addison-Wesley, 1973;

L. Reinders, H. Rubinstein and S. Yazaki, Phys. Reports C127 (1985) 1.

47. J. Schwinger, in ref. 446];

S. Güsken, J.H. Kühn and P.M. Zerwas, Phys. Lett. B155 (1985) 185.
48. I.I. Bigi, Y.L. Dokshitzer, V.A. Khoze, J.H. Kühn and P.M. Zerwas, Phys. Lett. B181 (1986) 157.

49. V.S. Fadin and V.A. Khoze, JETP Lett. 46 (1987) 525; Sov. J. Nucl. Phys. 48 (1988) 309.

50. J.M. Strassler and M.E. Peskin, Phys. Rev D43 (1991) 1500.

51. Y. Sumino, K. Fujii, K. Hagiwara, H. Murayama and C.-K. Ng, Phys. Rev. D47 (1992) 56.

52. M. Jeżabek, J.H. Kühn and T. Teubner, Zeit. Phys. C56 (1992) 653.

53. M. Jeżabek and T. Teubner, Zeit. Phys. C59 (1993) 669.

54. P. Igo-Kemenes, M. Martinez, R. Miquel and S. Orteu, in ref.[9], Part C, p.319.

55. Y. Sumino, in ref.[8], vol.II, p.439; Y. Sumino, PhD thesis, Univ.of Tokyo preprint UT-655 (unpublished);

K. Fujii, T. Matsui and Y. Sumino, KEK preprint 93-125.

56. M. Jeżabek and J.H. Kühn, Phys.Lett. B207 (1988) 91.

57. H. Überall, Phys. Rev. 119 (1960) 365;

R.W. Huff, Ann. of Phys. 16 (1961) 288.

58. H. Pilkuhn and F. Stäudner, Phys.Lett. A178 (1993) 156.

59. W. Mödritsch and W. Kummer, Wien Univ. preprint TUW-94-06, 1994.

60. W. Fischler, Nucl. Phys. B129 (1977) 157;

A. Billoire, Phys.Lett. B92 (1980) 343.

61. J.L. Richardson, Phys.Lett. B82 (1979) 272.

62. M. Jeżabek, J.H. Kühn and T. Teubner, in ref.[9], Part C, p.303.

63. I. Bigi et al., preprint CERN-TH.7171/94, hep-ph/9402360;

M. Benecke and V.M. Braun, preprint MPIPTh/94-9, hep-ph/9402364.

64. K.-I. Hikasa and Y. Sumino, private communication.

65. M. Jeżabek and J.H. Kühn, Phys.Lett. B316 (1993) 360.

66. B. Grządkowski, P. Krawczyk, J.H. Kühn and R.G. Stuart, Nucl. Phys. B281 (1987) 18.

67. R.H. Guth and J.H. Kühn, Nucl. Phys. B368 (1992) 38.

68. V.S. Fadin and O.I. Yakovlev, Sov. J. Nucl. Phys. 53 (1991) 1053. 